\title{
PRESENTACIÓN
}

\section{Irving A. Leonard Notable Hispanoamericanista Norteamericano}

Irving A. Leonard ocupa un lugar sobresaliente entre los hispanoamericanistas norteamericanos de este siglo, en rango equivalente a los más distinguidos de Europa y de la América hispánica. Por la cronología y por los méritos de su obra pertenece a la que debemos designar como la pioneer generation en los estudios de la cultura, la historia y las letras de Nuestra América en los Estados Unidos de Norteamérica. Encabezan esa legión de investigadores y críticos nombres hoy venerables como J.D.M. Ford, fundador, en el primer cuarto de este siglo, de un centro para estudios latinoamericanos en la Universidad de Harvard, Alfred Coester e Isaac Goldberg, autores de las primeras historias panorámicas de la literatura de Iberoamérica vista como una unidad cultural y artïstica, y los continuadores del Instituto Internacional de Literatura Iberoamericana-Herman E. Hespelt, John E. Englekirk, John Crow, John T. Reid y el mismo Leonard-, quienes prepararon el muy difundido Outline History of Spanish American Literature (1941) y su complemento textual An Anthology of Spanish American Literature (1946). En lo que va del siglo la lista de estudiosos dedicados a Iberoamérica ha crecido en forma que podríamos calificar de fabulosa. Estados Unidos se ha convertido en el primer centro hispanoamericanista del mundo (véase el National Directory of Latin Americanists (Washington: Library of Congress, 1966), no sólo por la cantidad y calidad de sus scholars, sino también por sus centros de investigación, bibliotecas de universidades e instituciones públicas y privadas, becas de estudio, medios de difusión y traducciones de textos. Puede decirse que no hay un especialista en el mundo que se precie de algún respeto en los estudios iberoamericanos que no haya venido o desee venir a estudiar o a enseñar e 
investigar en USA. Hoy la labor de los investigadores y críticos norteamericanos comparten con los alemanes, ingleses, franceses, españoles, italianos, etc. la responsabilidad de una tarea fecunda, seria e imprescindible, junto a hispanoamericanos que cumplen igual función en sus respectivos paises o trasladados y establecidos en los E.U. $\mathrm{Y}$ así, al lado de un Leonard, un Keniston, un Leavitt, un Rea Spell, hay un Pedro Henríquez Ureña, inspirado por Ford, doctorado en Minnesota y primer hispanoamericano que ocupó la famosa cátedra Charles Eliot Norton de Harvard. Como el maestro dominicano, también estudiaron y ejercieron cátedra de literatura hispanoamericana en universidades de este paǐs otros hispanoamericanos dignos de honrosa memoria, como Arturo Torres Rioseco, M.P. González, C. García-Prada, J.J. Arrom y muchos más. Un día tendremos que hacer la historia de esta falange de hispanoamericanistas norteamericanos y europeos e hispanoamericanos que echaron las bases de la más sólida investigación crítica para mejor comprender la historia, las letras, las artes y el pensamiento de la(s) otra(s) América(s). Y nos admiraremos de lo que ha contribuido, por ejemplo, E.K. Mapes al conocimiento del modernismo (Dario y Gutiérrez Nájera, sobre todo), Leavitt y Boyd Carter al de las revistas y periódicos, R.H. Hays, Dundas G. Craig y Dudley Fitts al de la poesía, W.R. Crawforf, J.L. Tate y S. Lipp al del pensamiento y la evolución de las ideas, D. Ratcliff, R. Spell y K. Schwartz al de la novela. La lista de nombres valiosos es inmensa y no es mi propósito hacerle justicia ni siquiera a una mínima parte de ella. Queden los S. Waxman, A.P. Whitaker, A.C. Vilgus, C.K. Jones, R.L. Grismer, A.W. Phillips, I.Schulman, F. Dauster, etc., junto a R. Lida, E. Anderson Imbert, L. Leal, H. Rodrïguez Alcalá, E. Rodríguez Monegal, J. Alazraki y tantos otros, para mejor oportunidad. Nuestro propósito es hoy el de honrar a un maestro de maestros: IRVING A. LEONARD, fundador de muchas cosas imperecederas, pero sobre todo uno de los que pusieron los fundamentos del Instituto Internacional de Literatura Iberoamericana, que edita la Revista Iberoamericana, en la cual dejamos constancia de nuestra admiración y agradecimiento.

Irving A. Leonard nació en New Haven, el hogar de la Yale University, el 1 de diciembre de 1896-coincidencia: año de la publicación de Prosas profanas y de la universalización de nuestra literatura a través de la prosa y la poesía del Modernismo-, y en dicha universidad obtuvo título en Ciencias Naturales. Enseguida viajó al Japón, China y Filipinas, en cuya Universidad, en Los Baños, enseñó ingeniería matemática (1921-1922). De regreso a USA. ingresó a la 
universidad de California, en Berkeley, donde obtuvo su master en español en 1925 y su doctorado en historia en 1928. Hasta 1937 fue profesor en dicha universidad californiana, una de las mejores de este y de cualquier pais de la más alta cultura. En ese lapso obtuvo becas del American Council of Learned Society y de la Guggenheim Memorial Foundation, con las que viajó en calidad de investigador por Europa y países de la América Latina. Desde 1937 a 1940 sirvió como asistente director de la División de Humanidades de la Rockefeller Foundation en Nueva York. Con tal cargo volvió a viajar por paises de su especial interés como México, Argentina, Colombia, Chile y Perú. Volvió a la cátedra en 1940, ahora como profesor de la Brown University. En 1942 pasó a la Universidad de Michigan, en Ann Arbor, como profesor de historia y literatura de la América Hispánica. Allí fue chairman del Departamento de Lenguas Romances por el periodo de 1945-1951. En dicha Universidad formó sus mejores discípulos e hizo sus más valiosas investigaciones (véase el curriculum vitae en este mismo número), que le valieron el nombramiento en la cátedra, para él creada, de "D. Domingo Faustino Sarmiento University Professorship". Se jubiló en 1965 y desde esa fecha es "Emeritus Professor".

Leonard ha publicado obras que marcan hitos en la investigación de aspectos fundamentales de la historia literaria del periodo colonial hispanoamericano. Casi no hay autor importante de los siglos XVII y XVIII que no haya frecuentado, revisado, corregido y agregado algún aspecto de su vida o de su producción. Pero su mejor contribución la ha dado en la investigación de la vida y la obra del máximo enciclopedista de América, D. Carlos de Sigüenza y Góngora. Siguen en el orden de las contribuciones individuales los estudios que ha dedicado a Pedro de Peralta Barnuevo y a Sor Juana. En un orden más abarcador de la cultura histórica y documental, Leonard abrió una brecha definitiva para la valoración de la cultura de España en sus dominios de ultramar con Books of the brave, monumento a la precisión histórica y prueba de una verdad que desmiente el control estricto del paso de libros e ideas (es decir, de cultura) de la Península a las tierras descubiertas, conquistadas y colonizadas. Con Baroque Times in Old Mexico Leonard dio nuevos rumbos para la comprensión del barroco hispánico, sobre todo el de la Nueva España, y ningún estudioso puede prescindir de este libro. Sus ediciones del Voyage ... de Jorge Juan y Antonio de Ulloa, de los Documentos inéditos de Sigüenza y Góngora, del Descubrimiento $y$ conquista... de Bernal Díaz del Castillo constituye otro aspecto 
de sus preocupaciones en las que puede considerarse como iniciador: el de la publicación y fijación crítica de los textos básicos de la historia y las letras de esta parte del Nuevo Mundo. Pero su labor no queda ahî: maestro en la cátedra fue también guľa en la críticacrítica histórica y positiva-en artículos de revistas especializadas y en obras de conjunto más vastas y universales, como la Collier's Encyclopedia, la Encyclopedia of World Biography, la New Catholic Encyclopedia y el Handbook of Latin American Studies, de cuya sección "Literatura colonial" fue editor desde 1935 a 1965.

Leonard ha modificado, enriqueciéndolo, el conocimiento que teníamos del barroco y del iluminismo colonial, y, en general, de la cultura (historia,-ideas, artes) de los tres siglos de la dominación española en América, incluyendo la parte española de los Estados Unidos. Sus aportaciones favorecen tanto a España como a sus hijas de allende el océano. Principalmente historiador, con un método de probidad documental insuperable, su visión de investigador e intérprete fue ante todo intelectual y humanìstica. Su obra marca el paso del especialismo positivista que imperaba en los departamentos de lenguas romances, de filiación filológica alemana, a la de la historia como resurrección y recreación del pasado, según la escuela de Ranke, por ejemplo, tan bien aplicada en los E.U. por el historiador Bolton (Precisamente Leonard recibió el premio Bolton por uno de sus libros). En distintos lugares nos ha dejado escrita esta actitud y función del historiador y crítico literario, y ésa es, creamos, la mejor herencia que podemos apreciar hoy de su contribución hispanoamericanista. Para Leonard, la América ibérica existe como un cuerpo vivo, con sus materiales crudos y cocidos, de adentro y de afuera, que hay que sopesar, distinguir, seleccionar, ordenar. Y en ese cuerpo que recibe y actúa todo existe como un sistema de supuestos interrelacionados. En Books of the brave dice:

In the study of modern history an often subtle interaction between literature and events in human affairs, particularly since the invention of the printing press, is not fully appreciated. Fictional writings are not only the subjective records of human experience, but sometimes the unconscious instigators of the actions of men by conditioning their attitudes and responses. Often the works of imagination that were most influential in this respect at a given time and place are not the supreme creations of genius; they are frequently inferior manifestations of artistic expression which, because of special circumstances, sway the thoughts and emotions of 
their readers more profoundly. As a result, they sometimes alter the course of history or modify contemporary customs and manners.

No se puede negar la tradición herderiana de esta visión histórica, que fue reactivada en tiempos de Leonard por la escuela histórica de Oxford, en especial por James Bryce. Así la historia y las letras quedaban unidas como complemento necesario para entender las voces de los pueblos y el Zeitgeist. Se comprende que diga en Baroque Times in Old Mexico:

This account seeks only to give an impression of the cultural, literary, and intellectual aspects of a relatively neglected period of Mexican history. It is, indeed, a kind of mosaic composed of bits of incident and anecdote and of larger pieces of personalistic and customary detail; it is in itself a kind of Baroque design.

En una entrevista que le hizo su discippulo Robert G. Mead, Jr., Leonard puntualiza su concepto de la literatura de la siguiente manera:

Para mï, la literatura es una parte de la historia, de la historia subjetiva, si se quiere, pero de la historia. Si la historia "formal" se ufana de ser objetiva, la literatura, más honrada, admite su subjetividad. La verdad es una fusión de valores objetivos y subjetivos, y el verdadero entendimiento se alcanza sólo cuando se comprenden las relaciones entre las dos clases de valores. El "arte por el arte" está fuera de las corrientes centrales del río de la historia y contribuye poco a nuestro entendimiento; el "arte al servicio de un gran ideal humano" está más cerca y a veces forma una parte central de este río, y por eso es valiosǐsimo auxilio al entendimiento. Los poetas y prosistas creadores nos comunican los sentimientos de otros seres humanos, el historiador nos relata los hechos humanos; los dos aspectos están vinculados de un modo invisible a la vida humana. ${ }^{1}$

Desde luego, privilegiar lo historicista no implica determinismo cultural. Leonard está lejos de una posición a los Taine o Comte. Lo que quiere nuestro autor es salvar la prioridad de los referentes como

1. En Homage to Irving A. Leonard... Edited by Raquel ChangRodriguez and Donald A. Yates (Michigan State University, 1977), p. 8. 
fenómeno de cultura; para el historiador, el referente es la cadena de la serie histórica, pero no exige necesariamente una razón mecánica de causa-efecto. Lo que sí implica es la existencia sine qua non del sujeto protagonista del hecho cultural, una especie de héroe u hombre representativo, como querian Carlyle y Emerson. Por eso, la historia no es causa de la literatura, sino más bien su efecto. Leonard no olvida destacar la calidad subjetiva del hecho artïstico, en el que hace radicar la honestidad de la literatura. Esto, desde el punto de vista de la crítica literaria lo aleja de la poiesis como acto puro de creación, que para él (aunque no lo dice así) es una especie de falacy. Procedente de la escuela de la cultura alemana (acaso Dilthey, Windelband, Rickert) ve la historia y la literatura como vivencia, y la obra literaria no como resultado del procedimiento, como quiere algún formalista ruso, sino como algo que dice, que tiene la necesidad de decir. En esos contenidos halla Leonard la riqueza de la historia y la validez de la obra literaria. Pero no minimiza los valores artǐsticos; antes bien, al no considerar al texto como mero mecanismo de composición, privilegia la función cultural de los valores estéticos, su validez histórica, vivencial. Parafraseando a Ortega y Gasset, me escribe en una carta: "Me parece muy atinado aseverar que la literatura es la literatura y la circunstancia," lo cual quería recalcar que "hay lazos muy estrechos entre las letras, los escritores y el ambiente colonial"; "Sin prescindir de la evaluación estética de una obra, conviene tomar en cuenta la circunstancia; es decir, los factores históricos, personales y culturales, y aun los elementos sociológicos que la integran. Tiene este proceder-concluye en un texto que me ha enviado en febrero de 1978- un valor doble: el lector profundiza en el significado de la obra, aumentando así su interés en ella, y también logra compenetrarse más hondamente de la cultura que la produce". El tex to no como fin en el texto, sino en el contexto que lo produce. Barthes, un cierto Barthes, no aprobarìa este modo de ver la obra literaria, pero Leonard sería muy bien acogido entre los partidarios de la socio-crítica, tal como la entiende Edmond Cros, por ejemplo. $\mathrm{Y}$ he aquil otro modo de ser actual, de interesarnos por su mensaje siempre renovado. 